\title{
FT-Infrared Spectroscopic Studies of Lymphoma, Lymphoid and Myeloid Leukaemia Cell Lines
}

\author{
$\underline{\text { Jaspreet Babrah }^{1,4}}$, Keith P. McCarthy ${ }^{3}$, Richard Lush ${ }^{2}$, Adam D. Rye ${ }^{2}$, Conrad Bessant ${ }^{4}$ \\ Nicholas Stone ${ }^{1,4}$. \\ ${ }^{1}$ Biophotonics Research Group, Gloucestershire Royal Hospital, Gloucester, UK \\ Tel: 01452 395708, Email: j.babrah@medical-research-centre.com \\ ${ }^{2}$ Department of Haematology, Gloucestershire Royal Hospital, Gloucester, UK \\ ${ }^{3}$ Department of Histopathology, Gloucestershire Royal Hospital, Gloucester, UK \\ ${ }^{4}$ Cranfield Health, Cranfield University, Silsoe, UK.
}

\begin{abstract}
This paper presents a novel method to characterise spectral differences that distinguish leukaemia and lymphoma cell lines. This is based on objective spectral measurements of major cellular biochemical constituents and multivariate spectral processing. Fourier transform infrared (FT-IR) maps of the lymphoma, lymphoid and myeloid leukaemia cell samples were obtained using a Perkin-Elmer Spotlight 300 FT-IR imaging spectrometer. Multivariate statistical techniques incorporating principal component analysis (PCA) and linear discriminant analysis (LDA) were used to construct a mathematical model. This model was validated for reproducibility. Multivariate statistical analysis of FTIR spectra collected for each cell sample permit a combination of unsupervised and supervised methods of distinguishing cell line types. This resulted in the clustering of cell line populations, indicating distinct bio-molecular differences. Major spectral differences were observed in the 4000 to $800 \mathrm{~cm}^{-1}$ spectral region. Bands in the averaged spectra for the cell line were assigned to the major biochemical constituents including; proteins, fatty acids, carbohydrates and nucleic acids. The combination of FT-IR spectroscopy and multivariate statistical analysis provides an important insight into the fundamental spectral differences between the cell lines, which differ according to the cellular biochemical composition. These spectral differences can serve as potential biomarkers for the differentiation of leukaemia and lymphoma cells. Consequently these differences could be used as the basis for developing a spectral method for the detection and identification of haematological malignancies.
\end{abstract}

Keywords: lymphoid leukaemia cell lines, myeloid leukaemia cell lines, lymphoma cell line, haematological malignancies, Fourier transform infrared (FT-IR) spectroscopy, multivariate statistical analysis, principal component analysis (PCA), linear discriminant analysis (LDA).

\section{INTRODUCTION}

Fourier transform infrared (FT-IR) spectroscopy is a vibrational technique that monitors changes in biochemical composition of cells at a molecular level. Haematological malignancies manifest at single cell level by subtle biochemical changes, which are often not obvious in histopathological studies. The non-invasive FT-IR spectroscopic method has been proposed to overcome limitations of current "gold standards" providing a diagnostic tool for haematological malignancies without the need for staining. Automated, repetitive analyses can therefore be carried out at a low cost with the added advantage of utilizing small sample volumes. In addition, FT-IR spectroscopy can distinguish differences in the characteristics of diverse molecules by probing chemical bond vibrations. These molecular and sub-molecular profiles can be used to define and differentiate cell types. The appeal of these factors has forced the exploitation of FT-IR spectrometers that combine superior spectral sensitivity with powerful multivariate statistical analysis.

Diagnostic Optical Spectroscopy in Biomedicine IV, edited by Dietrich Schweitzer, Maryann Fitzmaurice, Proc. of SPIE-OSA Biomedical Optics, SPIE Vol. 6628, 66280R, @ 2007 SPIE-OSA · 1605-7422/07/\$18 
This permits essentially all of the relevant biochemical information buried in the spectrum to be usefully extracted.

FT-IR spectroscopy uses the mid-infrared (IR) part of the electromagnetic radiation $\left(4000-400 \mathrm{~cm}^{-1}\right)$ to obtain an IR spectrum. It is based on the principle that when a sample is interrogated with an IR beam, the functional groups within the sample absorb the IR radiation. This induces vibrational motions (bending, stretching, etc) of covalently bonded moieties. The IR absorptions, when plotted against the wavelength of the light, produce a unique "biochemical fingerprint" of the molecular species present in the sample ${ }^{1}$. If this molecular fingerprint is modified by a disease process, then FT-IR spectroscopy can be used to detect and monitor the disease processes. Particular absorptions are assigned to various functional groups in an attempt to extract biochemical information. In complex biological materials such as cells, the major IR absorption bands arise from $\mathrm{N}-\mathrm{H}, \mathrm{C}=\mathrm{O}, \mathrm{C}-\mathrm{H}$ and $\mathrm{P}=\mathrm{O}$ bonds; found in proteins (present in cytoplasm), lipids (originate in cell membranes) and nucleic acids (found in nucleus) ${ }^{2}$. The sum or the specific arrangement of the bands in the IR spectra can provide qualitative and semi-quantitative information. This can be used to distinguish between normal and cancerous cells or tissues.

To date FT-IR spectroscopy has proven to be a powerful analytical tool to probe the structure and confirmation of lipids, proteins and nucleic acids ${ }^{3}$ and also of complex biological materials such as tissues, body fluids and cell cultures ${ }^{4,5}$. The ability of FT-IR spectroscopy to detect changes in the chemical composition and morphology of cells led to its use in distinguishing differences among cell populations; for example, between normal and cancerous cells ${ }^{6,7}$ as well as characterisation of cancerous state using proliferative cell lines at various stages of the cell cycle ${ }^{8}$. Consequently, FT-IR spectroscopic study has become an active area of research in the field of haematology, particularly in detecting and monitoring characteristic changes in molecular compositions and structures that accompany the transformation from a normal to a cancerous state. There have been various studies of FT-IR spectroscopy into haematological malignancies, including studies of differentiation between normal leucocytes and leukaemia cells based on qualitative and quantitative differences at the DNA, protein and lipid levels ${ }^{9}$, 10; follow-up chemotherapy assessments to predict drug-sensitive or drug-resistant ${ }^{11-13}$, determination of apoptosis in leukaemia cells $^{14,15}$ and differentiation of clinically aggressive (high-grade) and clinically indolent (low-grade) lymphomas based on the nucleic acid absorbance ratio ${ }^{16}$.

Here we describe a novel method based on a combination of mid-FTIR spectroscopy of dry films with advanced multivariate statistical analysis. There are several reasons to favour this approach; only microliters of cell culture are required for measurement, the cultured cells are much more homogeneous with high sensitivity to certain key analytes. This report presents preliminary results obtained from the analysis of lymphoma, myeloid and lymphoid leukaemia cell lines based on spectral variations due to biochemical differences. The spectral data were subjected to both unsupervised and supervised methods of multivariate statistics, in particular principal component analysis fed linear discriminate analysis (PCA-fed LDA) to elucidate the spectral variations between the lymphoma, myeloid and lymphoid leukaemia cell lines. The enduring aim of this study is to demonstrate that multivariate statistical methods can differentiate between cell types, which may aid in the development of a diagnostic algorithm to analyse blood and bone marrow aspirate specimens.

\section{MATERIALS AND METHODS}

\subsection{CELL CULTURE}

Lymphoma (Karpas), lymphoid (REH and $A C V$ ) and myeloid (HL60 and Meg01) leukaemia cell lines were obtained from the Section of Haemato-Oncology, at the Institute of Cancer Research, Sutton, UK. REH and $A C V$ are derived from human lymphoid leukaemia, HL60 and Meg01 are derived from human myeloid leukaemia and Karpas 299 is derived from Hodgkin's lymphomas. The cells were grown as a suspension in 80-90\% RPMI 1640 medium containing $10-20 \%$ fetal bovine serum (FBS). Cells were maintained at $37^{\circ} \mathrm{C}$ in a humidified atmosphere with $5 \%$ $\mathrm{CO}_{2}$.

\subsection{SAMPLE PREPARATION}

Prior to spectroscopy, cells were washed and diluted (1:5) in cold, sterile saline $(0.9 \% \mathrm{NaCl})$ solution. The samples were pelleted by centrifugation at $3000 \mathrm{rpm}$ for $3 \mathrm{~min}$ to concentrate the cells. The pellet was re-suspended in $0.9 \%$ saline solution and the washing cycle was repeated twice more. Typically $50 \mu \mathrm{L}$ of the final diluted cell sample was 
transferred onto two $\mathrm{CaF}_{2}$ slides by Cytospin ${ }^{\mathrm{TM}}$ (1500 rpm for $5 \mathrm{~min}$ ), a method formerly developed for cytology. The aim of this technique is to produce a monolayer of cells. The slides were then allowed to air dry at room temperature for $30 \mathrm{~min}$ as thin circular disk films (diameter of 2-3 mm). The cell samples were examined by FT-IR spectroscopy and a transmission spectrum was acquired for the resulting films. This approach finesses the difficulties associated with strong water absorptions by simply eliminating water from the specimen. In addition to eliminating the spectral inference of water, this approach can provide better spectral resolution by the virtue of eliminating the water/solute interactions.

\subsection{SPECTRAL MEASUREMENT}

The infrared spectral data presented in this study were collected using a Perkin-Elmer Spectrum One/Spotlight FT-IR Imaging 300 System based at Gloucestershire Royal Hospital. This instrument is incorporated with a computerised microscope XYZ stage, visual image capture software and a highly sensitive, liquid nitrogen-cooled $16 \times 16$ element $\mathrm{HgCdTe}(\mathrm{MCT})$ focal plane array detector with a step scanning interferometer. Visual images of lymphoid, myeloid and lymphoma cell lines were collected via a CCD camera under white light LED illumination in transmission mode. The desired regions of the visual image map were selected and measured using a resolution of $8 \mathrm{~cm}^{-1}$ with a step size of $6.25 \mu \mathrm{m}$ and 128 scans per pixel (spp) to increase the signal-to-noise ratio. Absorption spectra were acquired in the wavenumber range of 4000 to $720 \mathrm{~cm}^{-1}$. Measurements of each cell type were repeated three times with subsequent sets (1-3) prepared at different times. Background scans were obtained from a region of no sample and ratioed against the sample spectrum each time.

\subsection{DATA ANALYSIS/PRE-PROCESSING}

Prior to multivariate statistical analysis, the absorption spectra were converted using PS convert (Perkin Elmer) to a more compact data format. MATLAB (version 6.1, (C) The MathWorks Inc.1994-2006) was used to pre-process the data. This aims to enhance the spectral features that incorporate information regarding biochemical composition and effectively to suppress or eliminate unnecessary artefacts. The dataset was cropped in spectral dimension (typically between $1800-800 \mathrm{~cm}^{-1}$ ). The truncated spectra were then smoothed using a 9-point Savitsky-Golay algorithm and normalized to minimise intensity variations between the spectral data set. All the spectra were then mean centred which calculates the average spectrum of the data set and subtracts that average from each spectrum. Peak positions were determined by using Spectrum/spotlight software (version 5.0.01). For each cell type, the mean spectrum was taken as an average of three different regions at various sites of the sample. Two different multivariate statistical analyses were employed in this study using MATLAB. This allows virtually all of the data present within the IR spectrum to be utilised. As the variation in spectral morphology seen between the different cell lines involves subtle differences across the range of wavenumbers, multivariate statistical analysis allows better separation of the cell line types.

Firstly, an unsupervised PCA was applied to the spectral dataset in order to compress data, without losing relevant information. It is a well-established method ideally suited to distinguish and visualise small, reoccurring spectral variations in large data sets. It was used to describe the major trends of variation in the data and to establish whether or not spectra of cells group into classes due to cell type. Therefore, it can be used as a first step for classification of the 5 cell line groups. In this study, it was employed to generate pseudocolour maps of the significant principal component scores for each cell sample. ANOVA was performed to determine which PCs best differentiate between the cell line groups. The differences in the spectra across each cell sample were used for the clustering of cell line populations. Spectra from different cell line types were assigned different colours. The colour coded scatter plots enabled the natural separation of the data to be visualized.

The second methodology was a supervised LDA, where the number of groups (cell line class identity) is dictated rather than discovered during the analysis. The scores for a specified number of principal components are used as the input data for LDA. For the purpose of this study, LDA was used to, without altering the data contained within the spectra emphasize the clustering of different cell line types following PCA. LDA for the different cell line types involves finding a linear discriminate function (LDF) that maximises the separation in discriminate space between different cell line types, whilst minimising the separation within each group. The combination of these two statistical methods referred to as PCA fed LDA was used to create algorithms for the different cell line types. The constructed algorithm was tested for its ability to correctly predict the cell line type. The results are presented as the proportion of spectra correctly predicted or classified into a group. 


\section{RESULTS AND DISCUSSION}

In the present study, FT-IR spectroscopy of pure lymphoma (Karpas), lymphoid ( $R E H$ and $A C V$ ) and myeloid (HL60 and $\mathrm{Meg} 01$ ) cell lines was carried out. We demonstrated reproducibility of the spectroscopic and multivariate statistical methods to distinguish between cell line types. Figure 1 shows white light image and pseudocolour maps, of total intensity at each point, in transmission mode of REH cell line. The pseudocolour maps shows absorbencies of varying intensities, where blue indicates low and red indicates high absorbance values. These were produced for all five cell lines by using a motorized XYZ stage to collect spectra, in an XY co-ordination, from a selected area. This way each pixel region on the map represents the spectrum for that point. The variation of spectral differences across a sample was established by calculating principal components. Each PC is composed of PC loads, which are the spectral components and PC scores, which relates to the abundance of that spectral component in the sample. Figure 2 show the PC scores and loads of the first 10 principal components for $R E H$. As the number of principal components increase more subtle variations can be seen. The score map from the second principal component was used to identify the major differences between spectra across the map. Peak assignment of the loads demonstrates further variation in the distribution of lipids, proteins and nucleic acids. Averaged spectra from the three regions of interest were plotted for comparison and identification of spectral differences as shown in figure 3 . The spectrum in figure 4 is the mean representative spectrum for the $R E H$ cell line highlighting the prominent absorptions. Pseudocolour maps as well as their representative mean spectra were obtained for each cell type, but have not been presented in this paper due to the sheer number of data sets. Figure 5 represents the mean spectra from three sets of measurements obtained for each cell line (REH, ACV, HL60, Meg01 and Karpas) as representative spectra for further spectral analysis. Figure 5 also shows the peak intensity variations between the mean IR spectra of the five cell lines groups.

The IR spectra of cell lines are complex, with hundreds of data points due to the sheer number of contributing biomolecules and therefore contain many overlapping bands. In addition, the spectral homogeneity of spectra from cells belonging to similar lineage makes the visual inspection of individual spectra nearly impossible, and requires the use of methods of multivariate analyses for a meaningful interpretation. The subsequent results from these evaluation techniques are discussed in sections 3.2 and 3.3.

A

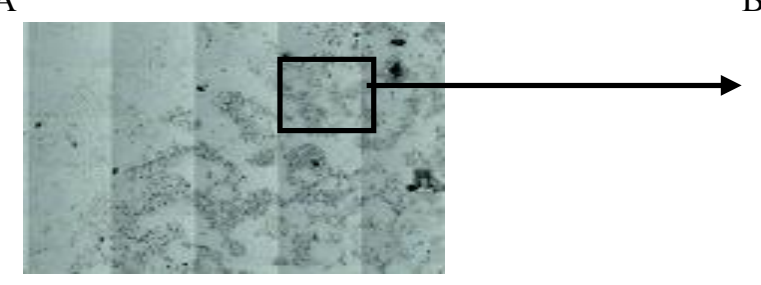

B

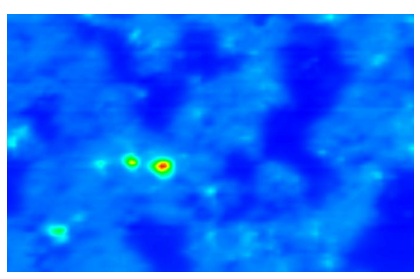

Figure 1: a) White light image of $R E H$ cell line and b) pseudocolour map of $R E H$ cell line in transmission mode. Black box highlights the region of interest.

A

PC Loads

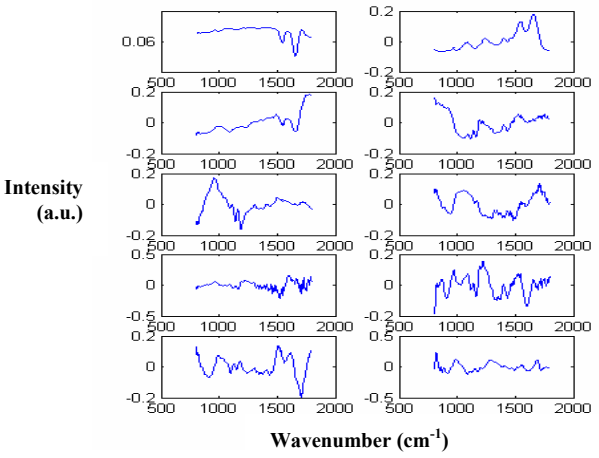

B

PC scores

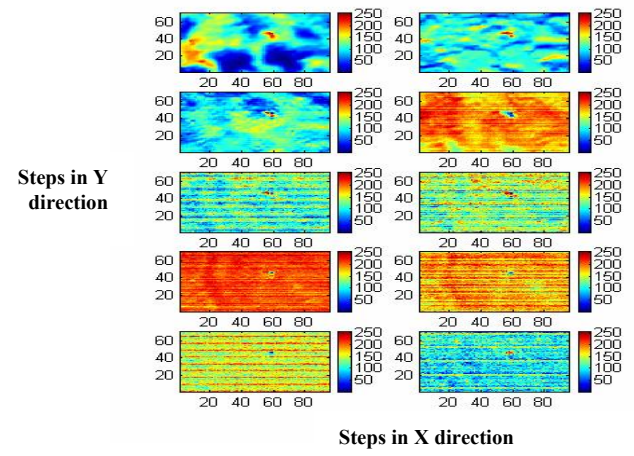

Figure 2: First 10 PC loads and corresponding pseudocolour score maps for the $R E H$ cell line. 

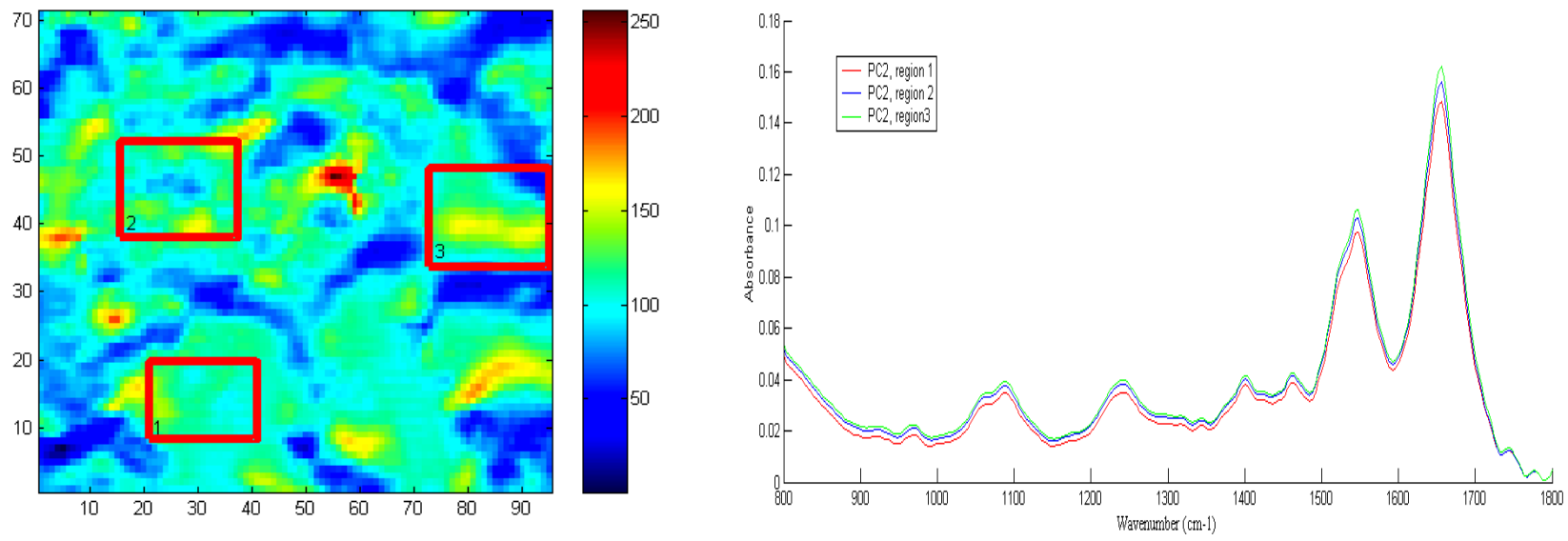

Figure 3: a) PC 2 score map with three selected regions of interest marked, b) mean IR spectra from three selected areas, region 1 (red line), region 2 (blue line) and region 3 (green line).

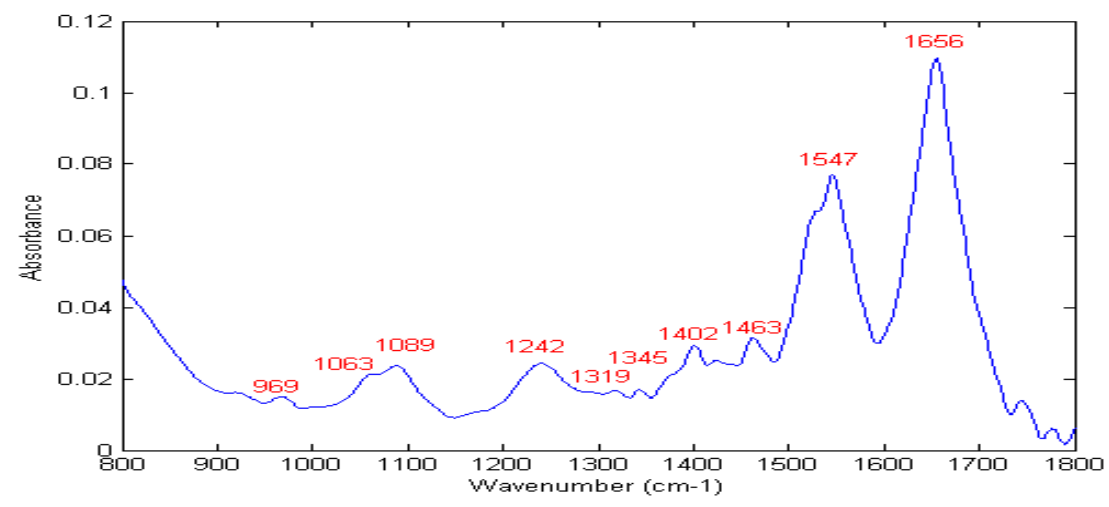

Figure 4: Representative mean spectra for REH cell line indicating major bio-molecular constituents.

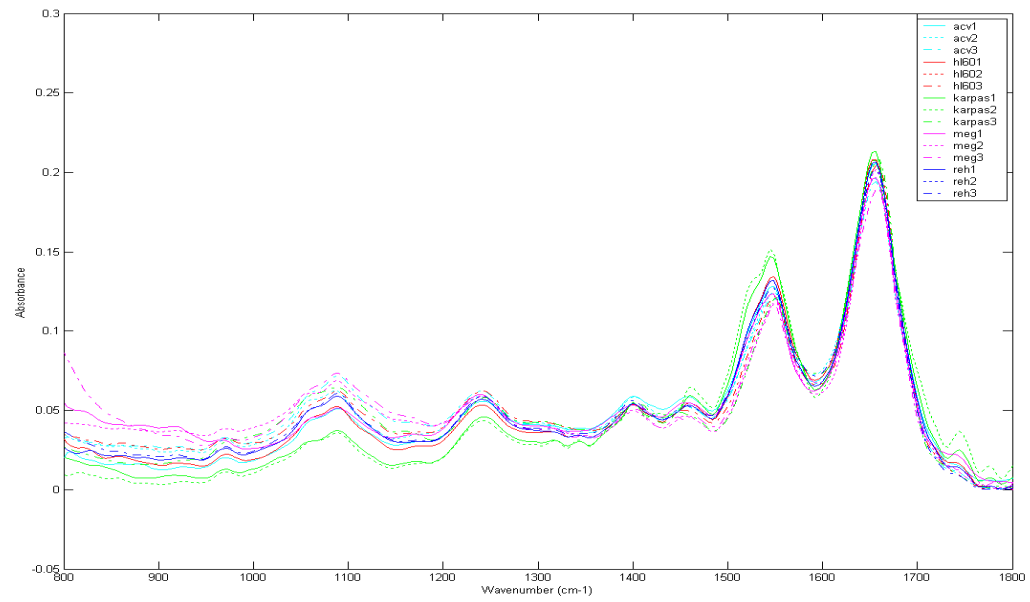

Figure 5: Mean normalised spectra of lymphoid ( $R E H$ and $A C V$ ), myeloid (HL60 and MegO1) and lymphoma (Karpas) cell lines from 3 sets of measurements. Set 1 is indicated by solid line $(-)$, set 2 is indicated by dotted line $(\cdots \cdots \cdot \cdots)$ and set 3 is indicated by dash-dot line (- - - ). 


\section{1: PEAK INTENSITY ANALYSIS}

The IR absorption spectra acquired in the region $800-1800 \mathrm{~cm}^{-1}$ using the methods described in section 2.3 were consistent with those described in various publications ${ }^{8-10}$. The IR spectra of cell lines show differences in the intensities of the peaks caused by differences in the bio-chemical constituents. This indicates considerable variation between the different cell line groups with subtle variation within the samples (figure 4 and 5). The most intensity variations are seen in the region of intensity $800-1300 \mathrm{~cm}^{-1}$ indicating major variations in the lipid and nucleic acid content of the cell lines. Figure 5 shows higher nucleic acid content in myeloid cell lines (e.g. Meg01) and lower amide I and amide II protein levels in lymphoma cell line (karpas). In comparison, lymphoma cell line has higher proteins levels and lower nucleic acid content.

Despite the minor spectral differences, the IR spectra of all cell lines are dominated by bands assigned to the absorption modes of proteins: the most intense is the amide I band, centred near $1656 \mathrm{~cm}^{-1}$, which corresponds to the $\mathrm{C}=\mathrm{O}$ stretching coupled to the $\mathrm{N}-\mathrm{H}$ bending and the $\mathrm{C}-\mathrm{H}$ stretching modes of peptidic bonds. It has been reported previously that the shape of the amide I band is influenced by the overall secondary structure of cellular proteins. In particular, the peaks assigned to $\alpha$-helices, $\beta$-sheets, turns and coils have absorption maxima between $1645-1662 \mathrm{~cm}^{-}$ ${ }^{1}, 1613-1637 \mathrm{~cm}^{-1}, 1662-1682 \mathrm{~cm}^{-1}$ and $1637-1645 \mathrm{~cm}^{-1}$ respectively. The amide II band at $1547 \mathrm{~cm}^{-1}$ arises from the vibrational modes that involve the C-N-H bending and C-N stretching of peptidic bonds. The amide III at $1242 \mathrm{~cm}^{-1}$ is contributed by proteins arising form C-N stretching and N-H bending. The peaks at $1400-1460 \mathrm{~cm}^{-1}$ are attributed to amino acids. The complex band pattern between $900-1300 \mathrm{~cm}^{-1}$ region represents the superimposed contributions of nucleic acids (DNA, RNA), carbohydrates and phosphates. In particular, the band centred at $1089 \mathrm{~cm}^{-1}$ is assigned to $\mathrm{C}-\mathrm{O}$ bending modes of saccharides (glucose and fatty acids) and the peak at $969 \mathrm{~cm}^{-1}$ form the $\mathrm{C}-\mathrm{C}$ stretching mode of (deoxy) ribose that is present in the DNA and RNA.

\section{2: PRINCIPAL COMPONENT ANALYSIS}

Multivariate analysis by PCA was initially used alone to classify the cell types for visual comparison. PCA allows the degree to which the algorithm groups spectra from the same cell line type, and separates the spectra from different cell line types to be represented visually using scatter plots. Scores of principal components (PC2 vs. PC4) were used for the plots with the position of each of the spectra marked on the plot. The separation of lymphoma and lymphoid and myeloid leukaemia cell lines produced is shown as two-dimensional plot in figure 6 .

As a result of strong similarities in their spectral signatures, the lymphoid leukaemia cell types (REH and $A C V)$ formed one cluster with overlapping spectra and the myeloid leukaemia cell types (HL60 and Meg01) produced the second cluster again with overlapping spectra. The third cluster belongs to the lymphoma (Karpas) cell line. PC scatter plots reveal good homogeneity of the spectra belonging to a particular lineage. This indicates prominent spectral variation between the different cell lines with very little intra-variability within the samples.

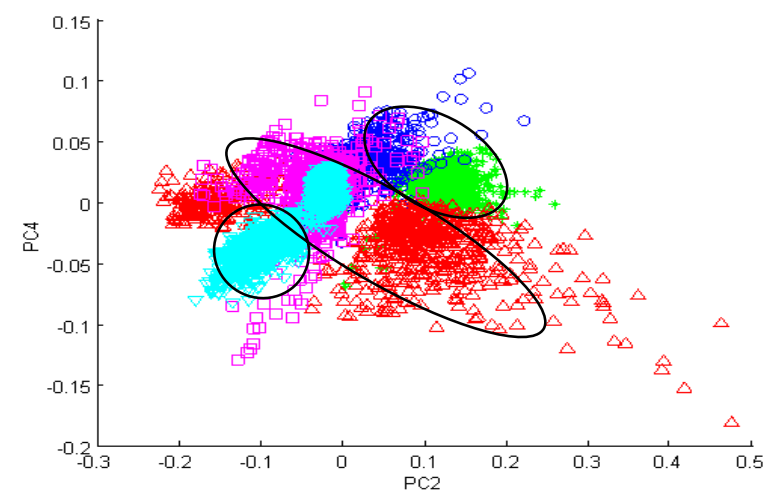

Figure 6: a) scores scatter plot (PC2 vs. PC4) represents clustering of lymphoid, myeloid and lymphoma cells lines. Each symbol represents one spectrum. Each symbol represents one spectrum. Lymphoid leukaemia cell lines $(A C V=$ red upward triangle and $R E H=$ magenta squares $)$, myeloid leukaemia cell lines $(H L 60=$ blue squares and $M e g 01=$ green stars $)$ and lymphoma cell line (Karpas $=$ cyan downward triangle). 


\section{3: LINEAR DISCRIMINANT ANALYSIS}

The five cell line group classification model generated using PC fed LDA demonstrated good separation of the spectra in linear discriminant space. LDA was performed to maximise the distance between the groups, whilst minimising the distance between members within each group. This is illustrated as the two-dimensional plot of the scores of the LD1 against the scores of LD4 in figure 7. The training performance of the model was $95 \%$. It also generated good sensitivity ranging from $94-99 \%$ and a specificity of $98-100 \%$ across the 5 cell line group classification model.

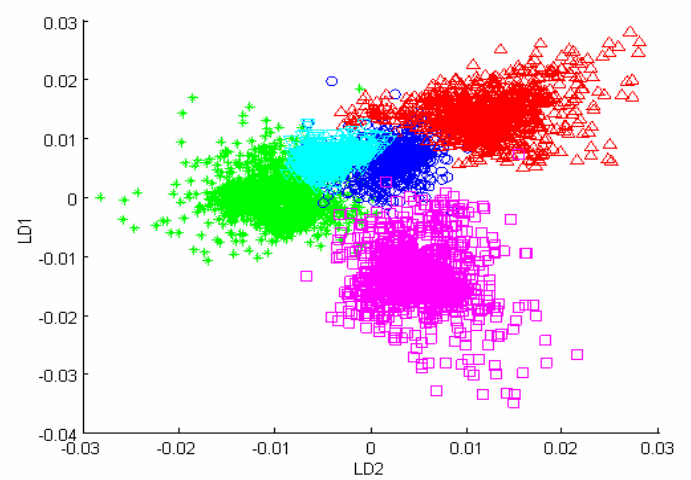

Figure 7: Scatter plot of the 5 cell line groups as generated by PCA fed LDA. Each symbol represents one spectrum. Each symbol represents one spectrum. Lymphoid leukaemia cell lines $(A C V=$ red upward triangle and $R E H=$ magenta squares), myeloid leukaemia cell lines $(H L 60=$ blue squares and $M e g 01=$ green stars $)$ and lymphoma cell line (Karpas= cyan downward triangle).

\begin{tabular}{|c|c|c|c|c|c|c|c|}
\hline \multirow{4}{*}{} & \multicolumn{7}{|c|}{ FT-IR Analysis } \\
\cline { 2 - 8 } & & ACV & HI60 & Karpas & Meg01 & REH & Total \\
\cline { 2 - 8 } & ACV & 1365 & 0 & 0 & 3 & 62 & $\mathbf{1 4 3 0}$ \\
\cline { 2 - 8 } Class & Hl60 & 59 & 1322 & 1 & 12 & 14 & $\mathbf{1 4 0 8}$ \\
\cline { 2 - 8 } & Karpas & 0 & 0 & 1293 & 6 & 10 & $\mathbf{1 3 0 9}$ \\
\cline { 2 - 8 } & Meg01 & 0 & 0 & 56 & 2000 & 41 & $\mathbf{2 0 9 7}$ \\
\cline { 2 - 8 } & REH & 118 & 0 & 0 & 1 & 1876 & $\mathbf{1 9 9 5}$ \\
\cline { 2 - 8 } & Total & $\mathbf{1 5 4 2}$ & $\mathbf{1 3 2 2}$ & $\mathbf{1 3 5 0}$ & $\mathbf{2 0 2 2}$ & $\mathbf{2 0 0 3}$ & $\mathbf{8 2 3 9}$ \\
\hline
\end{tabular}

Table 1: FT-IR five cell line group prediction model.

\begin{tabular}{|l|l|l|l|l|l|}
\hline Class & ACV & H160 & Karpas & Meg01 & REH \\
\hline FT-IR Sensitivity (\%) & 96 & 94 & 99 & 96 & 94 \\
\hline FT-IR Specificity (\%) & 98 & 100 & 99 & 99.7 & 98 \\
\hline
\end{tabular}

Table 2: Sensitivities and specificities achieved by the five group classification model as predicted by PCA fed LDA.

\section{CONCLUSION}

This study shows that small spectral differences exist between the infrared spectra of lymphoma, lymphoid and myeloid leukaemia cells. Irrespective of subtle spectral differences between cell types, it is possible to classify cells of different lineages via its spectral signature and unsupervised and supervised methods of multivariate statistics in particular PCA fed LDA. Spectral differences between the cell lines differ according to the cellular biochemistry, which can serve as potential biomarkers lymphoma, lymphoid and myeloid leukaemia. These initial results further indicate that this approach could be useful for early diagnosis of haematological malignancies. It also provides the added benefit that instrumentation does not require reagents to promote a visible change in colour. It also ensures that all IR compositional data is stored in a single data set, permitting the rapid extraction of information of components of interest. Further work will be carried out using additional cell lines to assess and compare sensitivity, specificity and reproducibility of FT-IR spectroscopy as a complementary diagnostic tool in the field of clinical haematology. With some improvement in the technique and the analytical approach, a very objective discrimination method can be developed. This could be promising for investigations based on fine needle aspirations and blood specimens. 


\section{REFERENCES}

1. Ellis D.I. and Goodacre R. "Metabolic fingerprinting in disease diagnosis: biomedical applications of infrared and Raman spectroscopy". The Analyst. Vol. 131, 875-885, 2006.

2. Mourant J.R., Gibson R.R., Johnson T.M., Carpenter S., Short K.W., Yamada Y.R and Freyer J.P. "Methods of measuring the infrared spectra of biological cells". Physics in Medicine and Biology. Vol. 48, 243-257, 2003.

3. Mantsch H.H. and McElhaney R.N. "Application of infrared spectroscopy to biology and medicine". Journal of Molecular Structures. Vol. 217, 347-362, 1990.

4. Erukhimovitch V., Talyshinsky M., Souprun Y., and Huleihel M. "Spectroscopic characterization of human and mouse primary cells, cell lines and malignant cells". Photochemistry and Photobiology. Vol. 74 (4), 446-451, 2002.

5. Delris G. and Petibois C. "Application of FT-IR spectroscopy to plasma contents analysis and monitoring". Vibrational Spectroscopy. Vol. 32, 129-136, 2003.

6. Cohenford M.A. and Rigas B. "Cytologically normal cells from neoplastic cervical samples display extensive structural abnormalities on IR spectroscopy: implication of tumour biology". Proc. Natl. Acad. Sci. USA. Vol. 95, 15327-15332, 1998.

7. Diem M., Boydston-White S. and Chiriboga L. "Infrared spectroscopy of cells and tissues: shinning light onto a novel subject". Applied Spectroscopy. Vol. 53, 148-161, 1999.

8. Boydston-White S., Gopen T., Houser S., Bargonetti J. and Diem M. "Infrared spectroscopy of human tissue. Infrared spectroscopic studies of myeloid leukaemia (ML-1) cells at different phases of the cell cycle". Biospectroscopy. Vol. 5, 219-227, 1999.

9. Benedetti E., Papineschi F., Vergammi P., Consolini R. and Spremolla G. "Analytical infrared spectral differences between human normal and leukaemic cells (CLL)". Leukaemia Research. Vol. 8, 483, 1984.

10. Schultz C.P., Lui K.Z., Johnston J.B., and Mantsch H.H. "Study of Chronic Lymphocytic Leukaemia Cells by FT-IR Spectroscopy and Cluster Analysis". Leukaemia Research. Vol. 20 (8), 649-655, 1996.

11. Lui K.Z, Schultz C.P, Johnston J.B., Mantsch H.H. "Comparison of Infrared spectra of CLL cells with their ex-vivo sensitivity (MTT assay) to chlorambucil and cladribine". Leukaemia Research. Vol. 21, 1125-1133, 1997.

12. Schultz C.P., Lui K.Z., Johnston J.B. and Mantsch H.H. "Prognosis of Chronic Lymphocytic Leukaemia from Infrared Spectra of Lymphocytes". Journal of Molecular Structures. Vol. 408/409, 253-256, 1997.

13. Ramesh J., Kapelushnik J., Mordehai J., Mose A., Huleihel M., Erukhimovitch V., Levi C and Mordechai S. "Novel methodology for the follow-up of acute lymphoblastic leukaemia using FTIR microspectroscopy". Journal of Biochemical and Biophysical Methods. Vol. 51 (3), 251-261, (2002).

14. Lui K.Z, Li. J, Kelsey S.M and Mantsch H.H. "Quantitative determination of apoptosis on leukaemia cells by infrared spectroscopy". Apoptosis. Vol. 6, 267-276, 2001.

15. Gasparri. F and Muzio. M. "Monitoring of apoptosis of HL60 cells by Fourier-transform infrared spectroscopy". Biochemical Journal. Vol. 369, 239-248, 2003.

16. Andrus G.L. and Strickland R.D. "Cancer Grading by Fourier Transform Infrared Spectroscopy". Biospectroscopy. Vol. 4, 37-46, 1998.

\section{ACKNOWLEDGEMENT}

The financial support for this research project by Leukaemia and Intensive Chemotherapy Charity (LINC) is gratefully acknowledged. Further support has been received from Biophotonics and Haematology teams at Gloucestershire NHS Trust. Author wishes to thank the hard work from Professor Mel Greaves and Sue Colman, of Institute of Cancer Research, Sutton for culturing the cell lines and Carol Baxter of Cytology Department, Gloucestershire Royal Hospital for the preparation of the slides. 